\title{
Pyridine derivative of the natural alkaloid Berberine as Human Telomeric G4-DNA Binder: a Solution and Solid-State study
}

Francesco Papi ${ }^{a}$, Carla Bazzicalupi ${ }^{a^{*}}$, Marta Ferraroni ${ }^{a}$, Giulia Ciolli ${ }^{a}$, Paolo Lombardi ${ }^{b}$, Asma Yasmeen Khan $^{{ }^{\dagger}}$, Gopinatha Suresh Kumarc, Paola Gratteri ${ }^{\mathrm{d}^{*}}$

\section{List of contents}

Details of used materials, Preparation of stock solutions

page 2

Circular dichroism spectroscopy

page 2

Spectroscopic titration experiments and binding data evaluation

page 3

Thermal melting studies

page 5

Isothermal titration calorimetry

page 5

X-ray diffraction analysis

page 7

Figure S1

page 4

Figure S2

page 4

Figure S3

page 5

Figure S4

page 6

Table S1

page 8

Figure S5

page 9 


\section{Materials}

The human telomeric sequences $d\left[T_{A} G_{3}\left(T_{2} A_{3}\right)_{3}\right]$ (Tel23) and $d\left[T A_{3} G_{2} A_{3} T\right]$ (Tel12) were purchased from Jena Bioscience (Jena, Germany) and used without further purification.

The semisynthetic ligand L is a proprietary compound, [ US Patent No. 8, 109 B2 to Naxospharma S.R.L., Italy, issued May 29, 2012] and was synthesized starting from commercial berberine chloride hydrate (ca. 17\% H2O) purchased from Shanghai Trust \& We, Ltd. (Shanghai, China) and 3-(pyridin-4-yl)propanal via a modification of an unusual enamine-aldehyde condensation performed on 7,8-dihydroberberine as previously reported .[ Chatterjee, S.; Mallick, S.; Buzzetti, F.; Fiorillo, G.; Syeda, T. M.; Lombardi, P.; Saha, K. D.; Kumar, G. S. RSC Adv. 2015, 5, 90632-90644]

\section{Preparation of stock solutions}

Solution studies. All biophysical experiments were conducted in $10 \mathrm{mM}$ potassium phosphate buffer of $\mathrm{pH}$ 7.4 that contained $100 \mathrm{mMKCl}$. pH measurements were made on a Sartorius PB-11 high- precision bench pH meter (Sartorius $\mathrm{GmBH}$, Germany) with an accuracy of $\mathrm{C} \pm 0.01$. The experiments were performed using glass distilled deionized water and analytical grade reagents. The chemicals were purchased from sigma and were of analytical grade. The buffer solution was filtered through Millipore filters (Millipore India Pvt. Ltd., Bangalore, India) of pore size $0.22 \mu \mathrm{m}$ before use. The concentration of the both the oligonucleotides (12 mer and 23 mer) was determined by absorbance using a molar absorption coefficient of $122800 \mathrm{M}-1 \mathrm{~cm}-1$ and $236500 \mathrm{M}-1 \mathrm{~cm}-1$ respectively. The molar absorption coefficient value of the oligonucleotide was determined using the nearest neighbour approximation model [Gray, D. M.; Hung, S. H.; Johnson, K. H. Methods Enzymol. 1995, 246,19-34]. The quadruplex structures were prepared by heating the samples to $363.15 \mathrm{~K}$, maintaining at this temperature for $10 \mathrm{~min}$ followed by slow cooling to room temperature ( 293.15 $\mathrm{K}$ ) and subsequent equilibration at $277.15 \mathrm{~K}$ for $48 \mathrm{~h}$.

Solid state studies. The compound $\mathrm{L}$ was dissolved in DMSO to a concentration of $20 \mathrm{mM}$. The oligonucleotides were dissolved to a concentration of either $1 \mathrm{mM}$ (Tel23) or $2 \mathrm{mM}$ (Tel12) in $50 \mathrm{mM} \mathrm{KCl}$ and $20 \mathrm{mM}$ potassium cacodylate buffer $(\mathrm{pH}=6.5)$ and then heated to $90^{\circ} \mathrm{C}$ for 15 minutes. The solutions were let cooling overnight to room temperature in order to induce quadruplex formation. The 1:1 adduct solution is obtained adding aliquots of the ligand DMSO solution to the targets. Milliq water from Millipore Water System (Millipore, USA) and analytical grade reagents were used for preparing crystallization screening solutions which were subsequently filtered through Sartorius-Stedim Biotech filters of $0.2 \mu \mathrm{m}$.

\section{Circular dichroism spectroscopy.}

Experimental. Circular dichroism spectra were recorded on a Jasco J815 spectropolarimeter (Jasco International Co., Ltd.) equipped with a Peltier cell holder and a temperature controller (model PFD425 L/15). Each scan was recorded at a scan speed of $200 \mathrm{~nm} / \mathrm{min}$ in the wavelength range 205-400 $\mathrm{nm}$ and at a bandwidth of $1 \mathrm{~nm}$, using rectangular cuvettes of volume $1000 \mu \mathrm{L}$ and a path length $1 \mathrm{~cm}$. A solution of quadruplex DNA $(6 \mu \mathrm{M})$ was titrated with increasing concentrations of L. Each spectrum was recorded 5 minutes after each addition to ensure complete complex formation. Four scans per sample were averaged to improve signal-to-noise ratio that was baseline-corrected and smoothed within allowed limits using the Jasco software of the unit.

Analysis. In the presence of $\mathrm{K}^{+}$solution, the Tel12 sequence gives a CD spectrum with a positive peak at 260 $\mathrm{nm}$ and a negative peak at $240 \mathrm{~nm}$ (Figure $1 \mathrm{~A}$ in the text, also reported below) which is typical of a parallel conformation. In the same condition, Tel23 adopts a hybrid-type mixed parallel/antiparallel stranded structure (3+1 hybrid structure) showing a positive peak at $290 \mathrm{~nm}$, a much smaller shoulder at $265 \mathrm{~nm}$ followed by a negative peak at $238 \mathrm{~nm}$ in its CD spectrum (Figure 1B in the text, also reported below).[ a) 
Vorličková M.; Kejnovská, I.; Sagi, J.; Renčiuk, D.; Bednářová, K.; Motlová, J.; Kypr, J. Methods 2012, 57 (1), 64-75; b) Randazzo, A.; Spada, G. P.; da Silva, M. W. Circular Dichroism of Quadruplex Structures. Top. Curr. Chem. 2013, 330, 67-86; c) del Villar-Guerra, R.; Trent, J. O.; Chaires, J. B. G-Quadruplex Secondary Structure Obtained from Circular Dichroism Spectroscopy. Angew. Chemie Int. Ed. 2018, 57 (24), 7171-7175; d) Luu, K. N.; Phan, A. T.; Kuryavyi, V.; Lacroix, L.; Patel, D. J. Structure of the Human Telomere in K+ Solution: An Intramolecular (3 + 1) G-Quadruplex Scaffold. J. Am. Chem. Soc. 2006, 128 (30), 9963-9970; e) Phan, A. T.; Kuryavyi, V.; Luu, K. N.; Patel, D. J. Structure of two intramolecular G-quadruplexes formed by natural human telomere sequences in K + solution. Nucleic Acids Res. 2007, 35 (19), 6517-6525.]

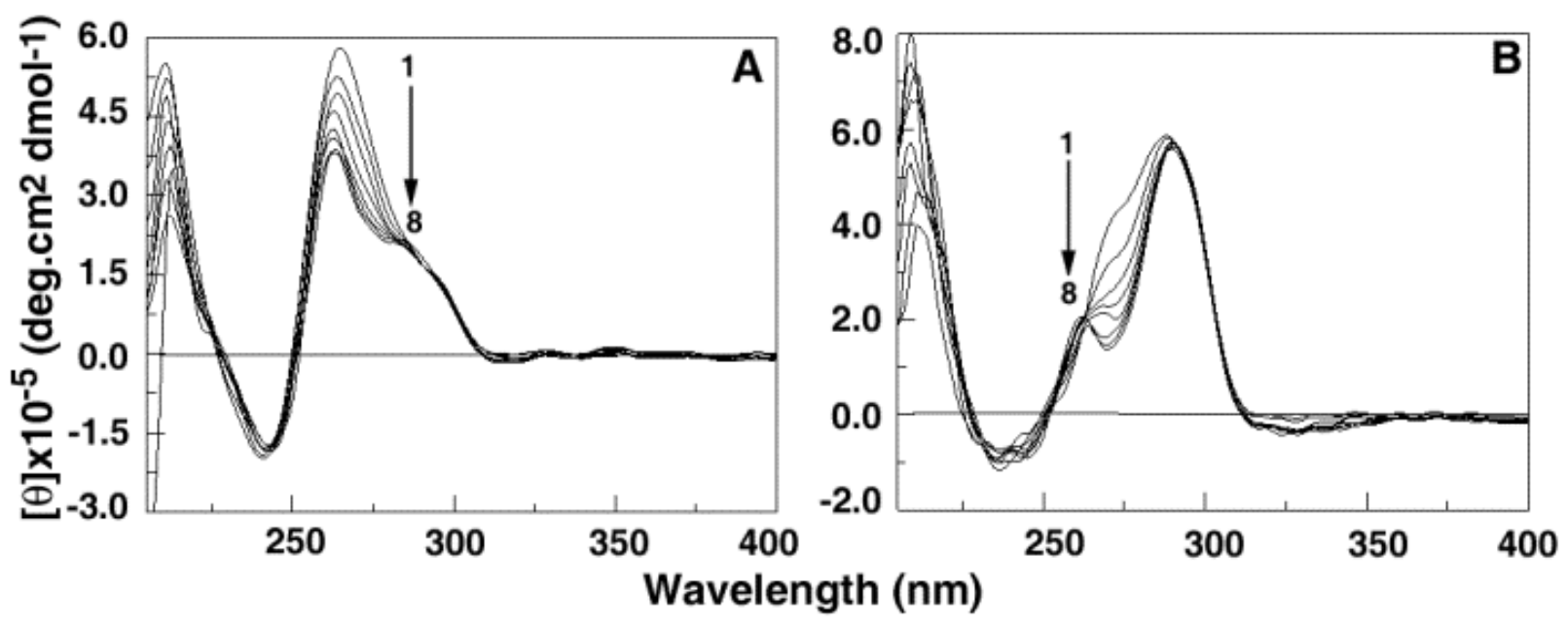

Figure 1. Circular dichroism spectra for the interaction of $L$ with $(A)$ Tel12 $(6 \mu \mathrm{M})$ with $0.0,3.0,6.0,9.0,15.0$, 21.0, 27.0 and $39.0 \mu \mathrm{M}$ (curves 1-8) and (B) Tel23 (6 $\mu \mathrm{M}$ ) with 0.0, 3.0, 6.0, 9.0, 15.0, 21.0, 33.0 and $45.0 \mu \mathrm{M}$ (curves 1-8).

\section{Spectroscopic titration experiments and binding data evaluation.}

Spectrophotometric titrations were performed on a Jasco V660 double beam double monochromator spectrophotometer (Jasco International Co., Hachioji, Japan). This experiment was performed at $293.15 \pm 1.0$ $\mathrm{K}$ by using thermoelectrically controlled quartz cuvettes following the standard protocols developed in our laboratory and described in detail previously.[ a) Giri, P.; Kumar, G. S. Arch. Bio-chem. Biophys. 2008, 474 (1), 183-192; b) Paul, P.; Kumar, G. S. RSC Adv. 2014, 4, 25666-25674] Steady state fluorescence measurements were performed on a Shimadzu RF-5301 PC fluorescence spectrometer (Shimadzu Corporation, Kyoto, Japan) in fluorescence free quartz cuvettes of $1 \mathrm{~cm}$ path length as previously described.[ Paul, P.; Kumar, G. S. RSC Adv. 2014, 4, 25666-2567420] The excitation wavelength of $L$ was $340 \mathrm{~nm}$ and an excitation and emission band pass of $5 \mathrm{~nm}$ was maintained during the measurements. The sample temperature was maintained at $293.15 \pm 1.0 \mathrm{~K}$ using an Eyela Uni Cool U55 water bath (Tokyo Rikakikai Co. Ltd., Tokyo, Japan). Binding data obtained from spectral titrations were converted into Scatchard plots ( $\mathrm{r} / \mathrm{Cf}$ versus $\mathrm{r}$ ) and were analysed using the cooperative McGhee-von Hippel equation as described previously.[ Paul, P.; Kumar, G. S. RSC Adv. 2014, 4, 25666-2567420, McGhee, J. D.; von Hippel, P. H. J. Mol. Biol. 1974, 86 (2), 469-489] 


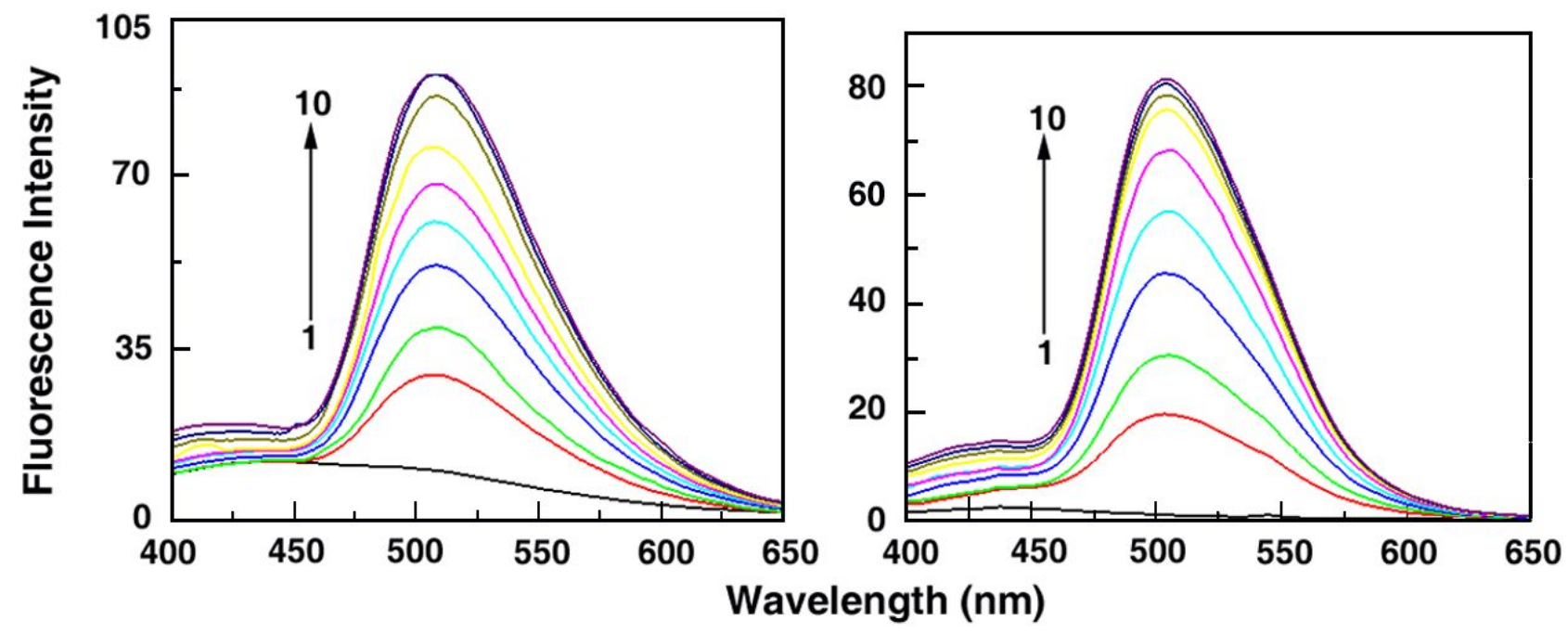

Figure S1. Representative fluorescence spectra of $L(1.0 \mu \mathrm{M})$ in the presence of (left) 0.0, 1.0, 2.0, 3.0, 4.0, 5.0, 7.0, 8.0, 10.0, and $12.0 \mu \mathrm{M}$ of Tel12 quadruplex DNA (curves 1-10), and (right) treated with 0.0, 1.0, 2.0, 4.0, 6.0, 8.0, 10.0, 12.0, 14.0, and $16.0 \mu \mathrm{M}$ (curves 1-10) of Tel23 quadruplex DNA.
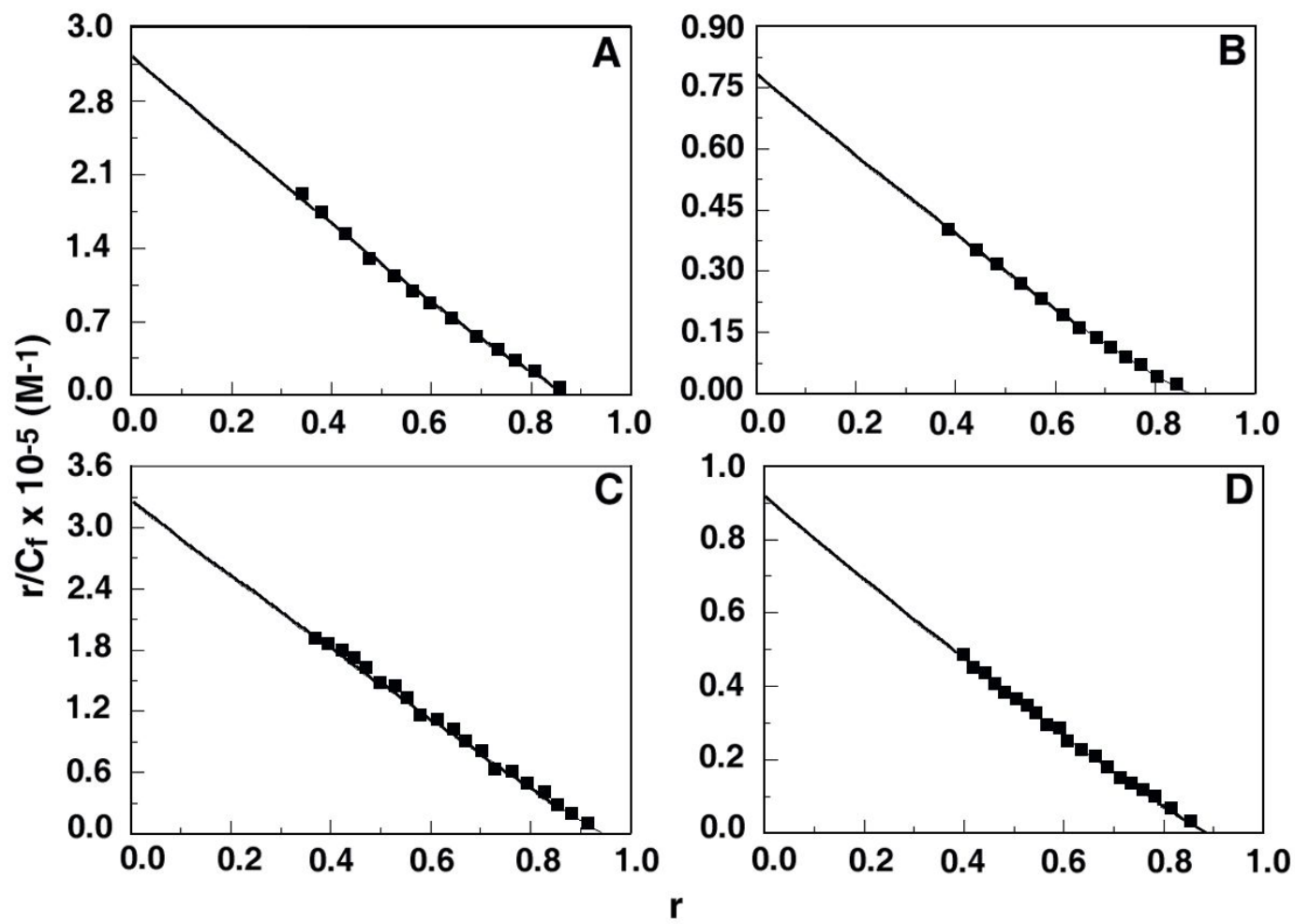

Figure S2. Representative Scatchard plots of each complexation. The plots were fitted and analyzed with a noncooperative model of McGhee and von Hippel.The solid lines represent the nonlinear least square best fit of the experimental points to the neighbour exclusion model. 


\section{Thermal melting studies}

Experimental. Thermal melting experiment was performed on a Shimadzu Pharmaspec 1700 unit (Shimadzu Corporation, Kyoto, Japan) equipped with a Peltier controlled Shimadzu thermoprogrammer (TMSPC-8) model accessory as described in details previously.[ Islam, M.; Pandya, P.; Kumar, S.; Kumar, G. S. Mol. BioSyst. 2009, 5, 244-254] This experiment was performed using Teflon capped 8 chambered micro optical cuvettes of $1 \mathrm{~cm}$ path length. The absorbance of quadruplex oligo samples (Tel12 and Tel23) and their [ligand]/[oligonucleotide](D/Ps)complexes of different molar ratios was monitored at $260 \mathrm{~nm}$ while raising the temperature at a rate of $0.5 \mathrm{~K} / \mathrm{min}$. The melting temperatures $(\mathrm{Tm})$ were obtained from the midpoint temperature of the unfolding profile.

Analysis. Thermal melting studies were performed by optical methods in order to gain further knowledge about the effect of the derivative on the stability of quadruplex structure upon complexation. An increase in melting temperature indicates an enhanced stability of the quadruplex upon interaction with the ligand. The melting profiles of the Tel12 and Tel23 quadruplex structure in the absence and presence of $L$ are shown in Figure S3A and S3B, respectively. Tel12 melted at $317.15 \mathrm{~K}$ while Tel23 melted at $336.15 \mathrm{~K}$ in the absence of the derivative; conversely, the Tel12-L complex melted at $332.15 \mathrm{~K}$ while that with Tel23 melted at $338.15 \mathrm{~K}$. Thus, a greater stabilization was obtained from the interaction of the alkaloid with Tel12 ( $\Delta \mathrm{Tm}$ increment of $15 \mathrm{~K})$ in comparison to Tel23 ( $\Delta \mathrm{Tm}$ increment of $2 \mathrm{~K}$ ) at 1:1 molar ratio.

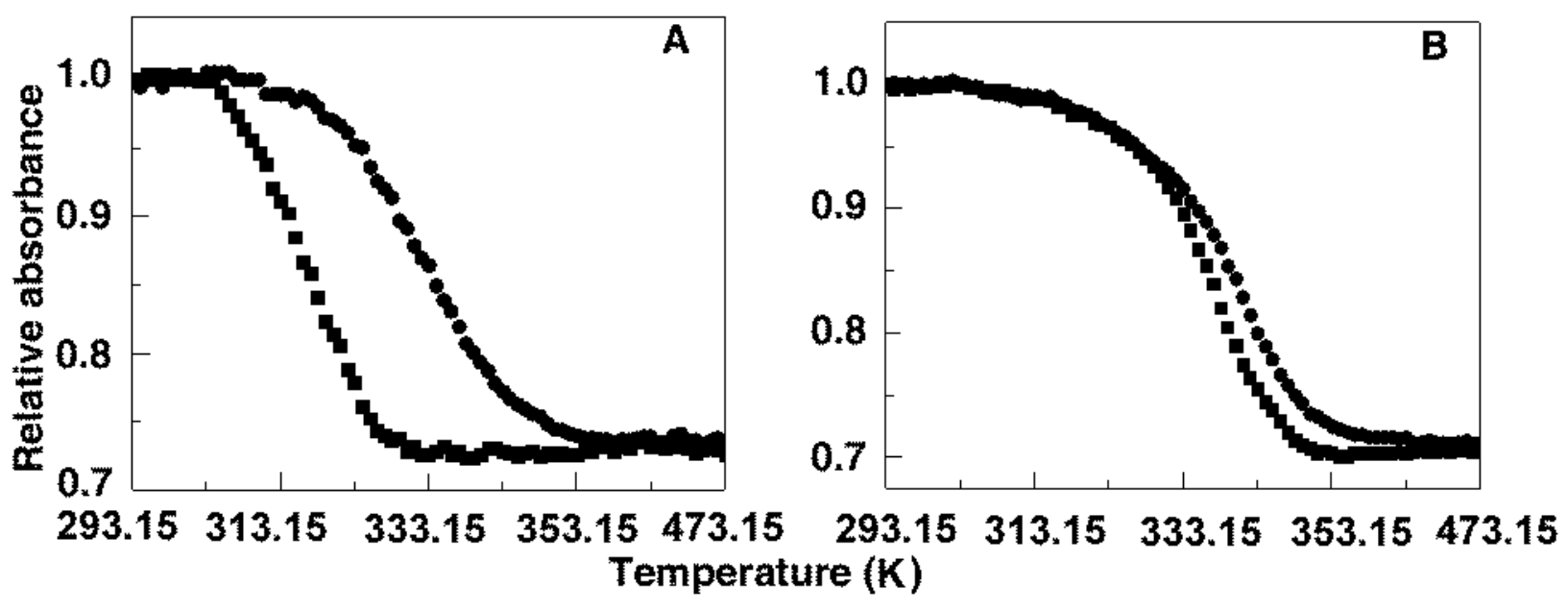

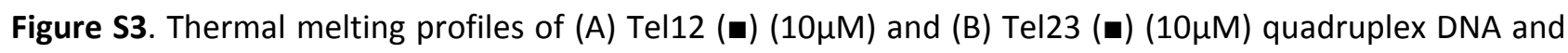
their respective complexes with $\mathbf{L}(\bullet)$

\section{Isothermal titration calorimetry.}

Experimental. Isothermal titration calorimetry (ITC) experiments were performed on a VP-ITC microcalorimeter (MicroCal, Inc., Northampton, MA, USA now Malvern Instruments Pvt. Ltd., Malvern, UK) to study the binding interaction of both the sequences of telomeric quadruplex DNA with L at $298.15 \mathrm{~K}$. The buffer solution was degassed prior to preparing the samples in order to prevent air bubble formation during titration. Titrations were performed by injecting $L$ solutions from the rotating syringe into the isothermal chamber containing the preformed quadruplex solutions $(10 \mu \mathrm{M})$. The telomeric quadruplex solution was titrated by injecting $10 \mu \mathrm{L}$ aliquots of alkaloid solution from the syringe (for a total of 28 injections, 20-s duration each at 4-min intervals) into the calorimeter cell. The corresponding dilution study of each reaction was determined in parallel experiments by injecting the solution of the alkaloid at the same concentration into the buffer. The heats of dilution were subtracted from the corresponding heat involved in the binding 
experiments prior to curve fitting that yielded the corrected isotherm. These isotherms were analysed using the built-in Origin 7.0 software that showed that only one type of binding event has occurred i.e. "one set of sites" model yielded the best fit curve for the obtained data points. The analysis of the data further yielded the binding constant $(\mathrm{K})$ and the standard molar enthalpy of association. The standard molar Gibbs energy $\left(\Delta G^{\circ}\right)$ and the standard molar entropy $\left(\Delta S^{\circ}\right)$ were calculated using standard thermodynamic relations described previously.[ Islam, M.; Pandya, P.; Kumar, S.; Kumar, G. S. Mol. BioSyst. 2009, 5, 244-254, Hossain, M.; Kabir, A.; Kumar, G. S. J. Biomol. Struct. Dyn. 2012, 30 (2), 223-234.] The change in heat capacity ( $\triangle \mathrm{Cp}^{\circ}$ ) for the association of $L$ with the quadruplex was calculated from the slope of the plot of enthalpy change $\left(\Delta H^{\circ}\right)$ versus temperature.

Analysis. Figure S5 depicts the calorimetric profiles for the titration of the alkaloid into the Tel12 and Tel23 solution at $298.15 \mathrm{~K}$. The thermograms in both cases reveal negative peaks in the plot of power versus time (upper panels of Figure S4) indicating an exothermic binding reaction. The amount of heat liberated on addition of alkaloids was plotted against molar ratio of alkaloid to quadruplex DNA (Figure S4, lower panel). Thereafter the actual heat evolved after every injection was calculated after correcting the injection heats for the heat of dilution. Heat of dilution was determined in a separate but identical experiment by injecting alkaloid into buffer alone (Figure S4, top panels, curves offset for clarity)

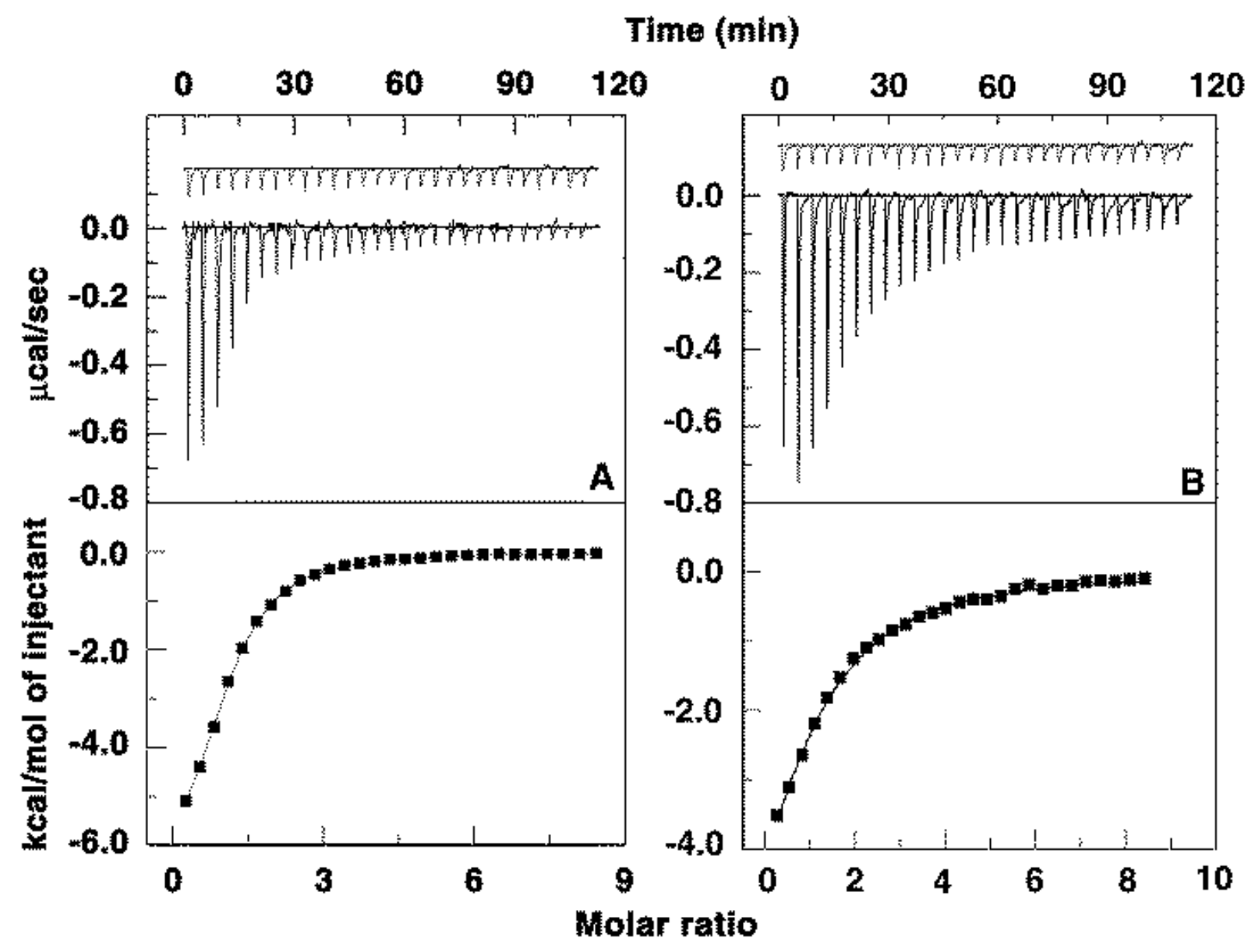

Figure S4. Isothermal titration calorimetry profiles for the titration of $\mathbf{L}$ with $(A)$ Tel12 $(10 \mu M)$ quadruplex DNA and $(B)$ Tel23 $(10 \mu \mathrm{M})$ quadruplex DNA at $T=298.15 \mathrm{~K}$. The top panels represent the raw data for the sequential injection of the drug into the quadruplex DNA solutions; the bottom panels show the integrated heat data after correction of heat of dilution against the molar ratio. The data points were fitted to one site model, and the solid lines represent the best fit data. 


\section{X-ray diffraction analysis.}

Crystallization Crystallization screenings were performed for $L$ and the human telomeric sequences Tel12 and Tel23. Crystallization experiments were set up by using the sitting drop crystallization method. Suitable crystals for X-ray analysis were obtained for the Tel12-L adduct by mixing $1 \mu \mathrm{L}$ of $0.001 \mathrm{M} \mathrm{G4} \mathrm{DNA/ligand} \mathrm{1:1}$ adduct to $1 \mu \mathrm{L}$ of a solution containing $0.05 \mathrm{M}$ potassium cacodylate buffer (pH 6.5), $0.05 \mathrm{M}$ Li2SO4, $0.05 \mathrm{M}$ MgSO4 and 20\% v/v iso-propanol and equilibrating against the same solution.[ Campbell, N. H.; Parkinson, G. N. Methods 2007, 43 (4), 252-263.]

Data collection and structure solution and refinement. Diffraction experiments on the crystals were performed at $100 \mathrm{~K}$, using as cryoprotectant the crystallization condition added with $30 \% \mathrm{v} / \mathrm{v}$ glycerol. Data were collected at the XRD-1 beamline (Elettra, Trieste) up to a maximum resolution of $1.60 \AA$, using $1.000 \AA$ wavelength X-ray. Data were integrated and scaled using the program XDS.[ Kabsch, W. Acta Cryst. 2010, D66, 125-132] The structure of the adduct was solved by molecular replacement using the program Molrep.[ Vagin, A. A.; Teplyakov, A. J. Appl. Cryst. 1997, 30, 1022-1025] The crystallographic coordinates of the Tel12-4 (Scheme 1 in the main text) adduct were used, as a search model, after deleting atoms belonging to ligand and solvent molecules (PDB accession number 5CDB).[ Ferraroni, M.; Bazzicalupi, C.; Papi, F.; Fiorillo, G.; Ortiz, L. M. G., Nocentini, A.; Scovassi, A. I.; Lombardi, P.; Gratteri, P. Chem. Asian J. 2016, 11 (7), 1107-1115] The Fo-Fc electron density maps showed a clear density for the drug molecule located on a 4-fold screw axis. The model was refined using the program Refmac5 from the CCP4 package.[ a) Murshudov, G. N.; Vagin A. A.; Dodson, E. J. Acta Cryst. 1997, D53, 240-255; b) Winn, M. D.; Ballard, C. C.; Cowtan, K. D.; Dodson, E. J.; Emsley, P.; Evans, P. R.; Kee-gan, R. M.; Krissinel, E. B.; Leslie, A. G. W.; McCoy, A.; McNicholas, S. J.; Murshudov, G. N.; Pannu, N. S.; Potterton, E. A.; Powell, H. R.; Read, R. J., Vagin, A. A.; Wilson, K. S. Acta Cryst. 2011, D67, 235-242] Thermal factors were treated as isotropic. Geometrical restraints for $L$ were generated by using the Grade web server[GRADE 1.2.12 (October 4th, 2016) - http://grade.globalphasing.org] and the ligand molecule was added manually into the electronic density maps. Manual rebuilding of the model was performed using the program Coot.[ Emsley, P.; Lohkamp, B.; Scott, W. G.; Cowtan, K. D. Acta Cryst. 2010, D66, 486-501] The crystal packing analysis was made by means of the Mercury program.[ Macrae, C. F.; Bruno, I. J.; Chisholm, J. A.; Edgington, P. R.; McCabe, P.; Pidcock, E.; Rodriguez-Monge, L.; Taylor, R.; van de Streek, J.; Wood, P. A. J. Appl. Cryst. 2008, 41, 466-470] Final coordinates and structure factors have been deposited with the Protein Data Bank (PDB accession number 5S15). Statistics of the data collection and refinement are reported in SI table S1. Structural figures were generated with the UCSF Chimera package.[ Pettersen, E. F., Goddard, T. D., Huang, C. C., Couch, G. S., Greenblatt, D. M., Meng, E. C. \& Ferrin, T. E. J. Comput. Chem. 2004, 25, 1605-1612] 
Table S1. Summary of Data Collection and Atomic Model Refinement Statistics. Values in parentheses are for the highest resolution shell.

\begin{tabular}{|c|c|}
\hline \multicolumn{2}{|l|}{ Data Collection } \\
\hline Wavelength (Å) & 1.000 \\
\hline Space group & $P 4_{1} 22_{1}$ \\
\hline Cell dimension (Å) & $a=b=41.25, c=68.60$ \\
\hline Limiting resolution (Å) & $35.35-1.70(1.81-1.70)$ \\
\hline Unique reflections & $6892(1061)$ \\
\hline $\mathbf{R}_{\text {sym }}(\%)$ & $8.1(26.1)$ \\
\hline Multiplicity & $23.0(21.7)$ \\
\hline Completeness overall (\%) & $99.3(97.7)$ \\
\hline$\langle 1 / \sigma(1)\rangle$ & $22.5(4.1)$ \\
\hline $\mathrm{CC}(1 / 2)$ & $99.9(99.7)$ \\
\hline \multicolumn{2}{|l|}{ Refinement } \\
\hline Resolution range (Å) & $35.35-1.70$ \\
\hline Unique reflections, working/free & $6173 / 702$ \\
\hline Rfactor (\%) & 26.57 \\
\hline Rfree(\%) & 29.35 \\
\hline r.m.s.d. bonds $(\AA ̊)$ & 0.010 \\
\hline r.m.s.d. angles $\left({ }^{\circ}\right)$ & 1.84 \\
\hline
\end{tabular}


Figure S5. Skeleton and 2Fo-Fc electron density map for $L$ contoured at $2.0 \sigma$ level

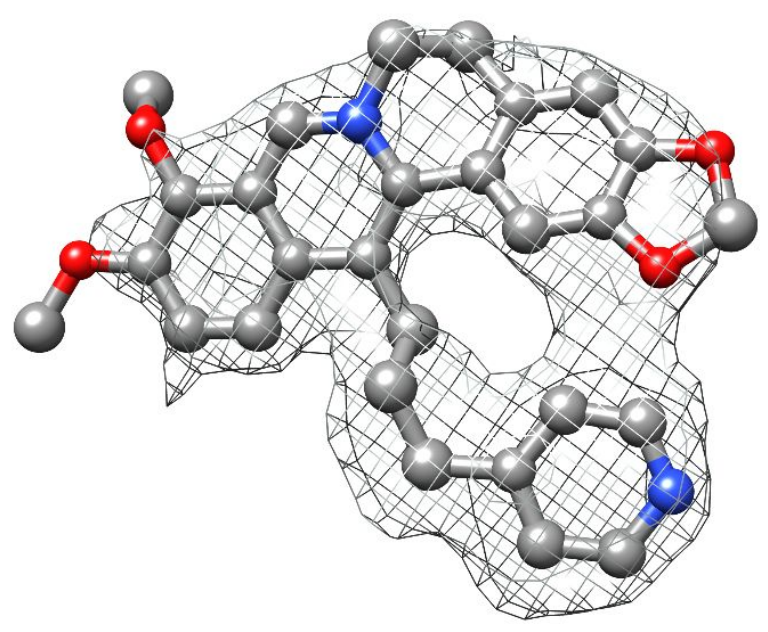

